\title{
Motivación al estudio de la Fisicoquímica en el Grado de Ciencia y Tecnología de Alimentos mediante el estudio de aplicaciones. Utilización para el desarrollo y evaluación de competencias transversales
}

\author{
Fernando Cardona $^{\text {a,b }}$, Lorena Atarés ${ }^{\text {a,c }}$
}

${ }^{a}$ Departamento de Tecnología de Alimentos. Escuela Técnica Superior de Ingeniería Agronómica y del Medio Natural. Universidad Politécnica de Valencia; ${ }^{b}$ fercarse@tal.upv.es; 'loathue@tal.upv.es

\begin{abstract}
It is known that for most students from other fields, the subject of physical chemistry is noticed as hard and difficult. This perception could be due, in addition to the intrinsic difficulty of the subject, to the apparent lack of application in the field (in our case Food Technology). In this project, examples related to food technology applications are introduced into the subject, as well as the possibility of do volunteer work related to them. The evaluation (self-evaluation) of the development of transversal skills and the degree of satisfaction and motivation of students was perform through anonymous surveys. The results show that students believe that the subject could be checkpoint of the skill "Problem analysis and solving" and that the performance of this works helps to develop several of these skills, coinciding almost entirely with the view of the professors. In addition, scores on the evaluation tests show a better average, especially in the part of problems resolution, for the student which have done the work than for which have not done.
\end{abstract}

Keywords: Physical Chemistry, Applications, Motivation, Transversal Skills

\section{Resumen}

Es conocido que para la mayoría de los alumnos de otros campos de trabajo, la asignatura de fisicoquímica se ve como dificil y poco amena. Esto podría ser debido, además de a la dificultad intrínseca de la asignatura, a la aparente falta de aplicación en el campo de trabajo de los alumnos (en nuestro caso Tecnología de Alimentos). En este proyecto, se introducen en la asignatura ejemplos relacionados con la Tecnología de Alimentos, así como la posibilidad de realizar trabajos voluntarios relacionados con estas aplicaciones. Además se evalúa (auto-evaluación), mediante encuestas anónimas, el desarrollo de competencias transversales y el grado de satisfacción y motivación del alumnado. Los resultados muestran que los alumnos opinan que la asignatura podría ser punto de control de la 
competencia "Análisis y resolución de problemas", y que la realización del trabajo ayuda a desarrollar varias de estas competencias, coincidiendo casi totalmente con la opinión del profesorado. Además, las calificaciones obtenidas en los exámenes muestran una mejor media, especialmente en la parte de problemas, en los alumnos que han realizado el trabajo optativo, respecto de los que no lo han hecho.

Palabras clave: Fisicoquímica, Aplicaciones, Motivación, Competencias Transversales

\section{1-Introducción}

Es conocida la dificultad que presenta para la mayoría de los alumnos de disciplinas que o son química o física, la asignatura de fisicoquímica (Mulop et al., 2012; Sokrat et al., 2014). Esta dificultad puede deberse, además de a la dificultad inherente que presenta la asignatura, a los conocimientos previos del alumno y al tiempo que es posible dedicar a resolver problemas prácticos durante el curso (Blanco-Vázquez, 2006). En general los alumnos del Grado de Ciencia y Tecnología de los Alimentos no entienden que esta materia esté relacionada con su campo, por lo que todavía les es más ardua de estudiar. Además, la transferencia de conocimiento que supone el establecer sinergias con otras disciplinas podría suponer una motivación adicional (Mainar-Fernández, 2009). Según Flores y Trejo (2003) se enfatiza demasiado en los métodos matemáticos para calcular propiedades, sin dejar claro antes el significado de los conceptos, lo que dificulta que los estudiantes puedan identificarlos y encontrar aplicaciones prácticas para los mismos en situaciones reales (Durán-Aponte y Durán-García, 2013). Esta situación puede conducir a la desmotivación del alumnado; tanto por la dificultad en la comprensión de los conceptos, como por la aparente falta de aplicabilidad de los mismos.

Así pues, la identificación de los conceptos químicos teóricos con situaciones reales en el campo de trabajo (enseñanza por proyectos "en contexto"), podría ayudar a la comprensión de los mismos, así como a la motivación de los alumnos (De Jong, 2006). Además, estas aplicaciones contribuirían a la identificación de la materia como relacionada con el campo de estudio elegido por el alumno, lo que sin duda haría aumentar su interés por la misma.

También es conocido que, mientras los alumnos con vocación por la química per se pueden recibir un enfoque de enseñanza más académico sin desmotivarse, los estudiantes interesados en otras disciplinas mejoran su motivación al recibir un enfoque más contextualizado (Eilks y Hofstein, 2015). En este último grupo estarían los alumnos de Ciencia y Tecnología de alimentos, ya que aunque puedan estar interesados en la química de alimentos no tienen una vocación química "pura".

En cuanto a las competencias transversales, tanto en la Universidad Politécnica de Valencia (UPV) como en otras universidades españolas y europeas, es necesaria la implantación de métodos para el desarrollo y la evaluación de las mismas a corto plazo, dentro de un proyecto estratégico de estas universidades, marcado por la adaptación al Espacio Europeo de Educación Superior. 
Si observamos varias definiciones de lo que es una competencia, observamos que siempre hacen referencia a que deben desarrollarse para situaciones lo más reales posible, lo más parecida a la situación laboral en la que se encontrará el individuo.

Definiciones de "Competencia" (García-García, 2009):

-Cinterfor/OIT, 1995: "La capacidad real para lograr un objetivo o un resultado en un contexto dado"

- Masterpasqua, 1991: "Características personales (conocimiento, habilidades y actitudes) que llevan a desempeños adaptativos en ambientes significativos"

-Consejo Federal de Cultura y Educación de Argentina: "Un conjunto identificable y evaluable de conocimientos, actitudes, valores y habilidades relacionados entre sí que permiten desempeños satisfactorios en situaciones reales de trabajo, según estándares utilizados en el área ocupacional"

- González y Wagenaar, 2003: Aquellas habilidades relacionadas con el desarrollo personal, que no dependen de un ámbito temático o disciplinario específico sino que aparecen en todos los dominios de la actuación profesional y académica.

En cuanto a los Tipos de Competencias, encontramos (García-García, 2009):

- Competencias Específicas (o técnicas)

- Relacionadas con sus conocimientos técnicos. Ej: Manejo avanzado de los equipos de medición acústica

- Competencias Transversales (o genéricas)

- Necesarias en un titulado con ese nivel académico, pero no necesariamente relacionadas con sus conocimientos técnicos. Ej: Capacidad de trabajo en equipo

Por lo tanto, una Competencia Transversal (CT) podría definirse como "la capacidad de lograr un objetivo o resultado en un entorno de aplicación real, que no está directamente relacionada con conocimientos técnicos". Así pues, parece lógico utilizar aplicaciones reales (enseñanza "en contexto") para desarrollarlas y para evaluarlas.

La evaluación por competencias nos obliga además a utilizar una diversidad de instrumentos y a implicar a diferentes agentes: tenemos que utilizar la observación, que puede acompañarse de registros cerrados o abiertos, y puede hacerse por parte del profesorado, por parte de los compañeros, por parte del propio estudiante (auto evaluación), o por todos ellos (Cano-García, 2008).

Las CT definidas por la UPV son:

1. Comprensión e integración.

2. Aplicación y pensamiento práctico.

3. Análisis y resolución de problemas.

4. Innovación, creatividad y emprendimiento.

2016, Universitat Politècnica de València

Congreso In-Red (2016) 
5. Diseño y proyecto.

6. Trabajo en equipo y liderazgo.

7. Responsabilidad ética, medioambiental y profesional.

8. Comunicación efectiva.

9. Pensamiento crítico.

10. Conocimiento de problemas contemporáneos.

11. Aprendizaje permanente.

12. Planificación y gestión del tiempo.

13. Instrumental específica.

Según la propia UPV (Preguntas frecuentes sobre CT)

(https://www.upv.es/entidades/ICE/info/U0702443.pdf):

"No se trata de impartir nuevas materias; se trata de incorporar actividades pensadas para el desarrollo de los contenidos de nuestra materia y que, a la vez, fuercen a los estudiantes a desarrollar y mejorar las habilidades ligadas a las diferentes competencias transversales $U P V$. Es decir, no se trata de impartir contenidos sobre las competencias transversales sino de idear actividades sobre nuestra asignatura que las trabajen."

En este sentido, este proyecto se propone introducir en la asignatura "Fundamentos Químicos para la Ciencia y Tecnología de los Alimentos" ejemplos relacionados con la Tecnología de Alimentos, así como la realización de trabajos voluntarios por el alumnado (que pueden exponer o no), relacionados con estas aplicaciones. Además el trabajo puede realizarse en grupos, permitiendo así en aprendizaje cooperativo, que ha demostrado su eficacia para el aprendizaje de esta materia (Méndez-Coca, 2012). La realización del trabajo supondrá para el alumno una aproximación de la asignatura al campo de trabajo de su grado, consiguiendo así que se evidencie la relación con este campo de trabajo, ayudando al entendimiento de los conceptos teóricos y motivando al alumno a su estudio.

También se ha postulado ya, que evaluar las CT implica plantear estrategias que se centren en la realización de actividades lo más cercanas posible al contexto profesional en el que los estudiantes se encontrarán al finalizar sus estudios, y con el apropiado nivel de complejidad (Fernández-March, 2010). Recordemos que la aplicación es el tercer nivel cognitivo de la taxonomía de Bloom (Bloom, 1956), por lo que no se podrá llegar al nivel máximo (evaluación) sin pasar por ella. Además, el aprendizaje basado en problemas, los simposios y los trabajos de investigación, han sido ya propuestos con éxito anteriormente como métodos para el desarrollo y evaluación de CT en Ciencias de Salud (Baños y Pérez, 2005).

Así pues, en este proyecto se pretende también evaluar el desarrollo de las CT definidas por la UPV con los trabajos voluntarios sobre aplicaciones de la Química Física a la Tecnología de Alimentos, especialmente en las CT: "Aplicación y pensamiento práctico", "Comprensión e Integración", "Trabajo en equipo y liderazgo" (si el trabajo se desarrolla en

(c) EY-NC-ND 2016, Universitat Politècnica de València 
grupo" y "Comunicación efectiva" (en el caso de que el trabajo se exponga en los seminarios de clase). Los alumnos se auto-evaluarán el grado de desarrollo de las competencias transversales, que el profesor comparará con sus observaciones. Las calificaciones obtenidas en la asignatura permitirán además comparar los resultados obtenidos entre los que han realizado el trabajo y los que no, con el objetivo de comparar su motivación al estudio de la materia.

\section{2-Objetivos}

-Introducir ejemplos de aplicaciones a la Ciencia y Tecnología de los Alimentos en la asignatura "Fundamentos Químicos para la Ciencia y Tecnología de los Alimentos" (Unidad Didáctica 2: Termodinámica).

-Evaluar el impacto que tiene la introducción de estos ejemplos de aplicaciones sobre la motivación de los alumnos en el estudio de la asignatura.

-Evaluar el desarrollo de adquisición de competencias transversales durante el desarrollo de los trabajos voluntarios sobre aplicaciones.

\section{3-Desarrollo}

Durante el curso académico 2014-2015 se planteó a los alumnos la posibilidad de realizar trabajos sobre aplicaciones de la Química Física a la Tecnología de Alimentos. Los trabajos eran voluntarios y servían para subir la nota final de la UD2 hasta un punto. Los alumnos tenían la posibilidad de hacerlo individual o en grupo (hasta un máximo de 3 participantes), así como de exponer el trabajo en clase o no, sabiendo que la exposición se tendría en cuenta a la hora de puntuarlo. El trabajo escrito incluye un resumen del contenido y una crítica sobre la aplicabilidad a la tecnología de alimentos. La exposición en forma de seminario incluye una exposición de máximo 15 minutos (entre varios si el trabajo se realiza en grupo) y un turno de preguntas de los compañeros de clase y el profesor.

Esta experiencia se llevó a cabo con alumnos de primer curso del Grado de Ciencia y Tecnología de Alimentos (ETSI Agronómica y del Medio Natural), durante el curso 20142015. La asignatura en la que se realizó la experiencia es "Fundamentos Químicos para la Ciencia y Tecnología de los Alimentos", una asignatura anual de 12 créditos, repartida en tres unidades didácticas. La UD2, Termodinámica, fue el contexto concreto de trabajo. La asignatura es punto de control de la CT "Instrumental específica".

2016, Universitat Politècnica de València

Congreso In-Red (2016) 


\section{4-Resultados}

\section{1-Muestra}

Del total de alumnos examinados (83), realizaron el trabajo 53 alumnos. Del total de alumnos matriculados, 45 realizaron la encuesta de auto evaluación de las competencias transversales, aunque algunos no la terminaron. De los 45 encuestados, 41 habían realizado el trabajo y 4 no. De los 41 que realizaron el trabajo, 15 no lo expusieron en clase, y 26 sí.

De los alumnos encuestados 32 (71.1\%) asistía siempre a clase, 9 (20.0\%) asistía regularmente, $3(6.7 \%)$ ocasionalmente y $1(2.2 \%)$ no asistía nunca a clase.

\section{2-Nota media}

Para todos los alumnos examinados, se calculó la nota media global (sobre 10, sin tener en cuenta la calificación extra obtenida por el trabajo), la media de la parte de teoría (3 puntos sobre 10) y de la parte de problemas (7 puntos sobre 10) (Figura 1). El análisis de la varianza ( $f$ de fisher) mostró que las muestras poseen varianzas diferentes (Tabla 1), por lo que se realizó una prueba estadística $t$ de student para muestras con varianzas diferentes (tabla 2). Todas las pruebas estadísticas se realizaron utilizando Microsoft Excel.

Se observa que los alumnos que han realizado el trabajo, presentan una mayor nota media final, y que la diferencia está principalmente en la parte de problemas (Figura 1). Las diferencias de medias son estadísticamente significativas ( $\mathrm{p}<0,005$; Tabla 2).

Además se observa que en general el porcentaje de alumnos con notas altas ( 7 y 8 ) es mayor para los alumnos que han realizado el trabajo, siendo especialmente evidente en el caso de notas mayores o iguales a 8 , donde todos los alumnos realizaron el trabajo (Figura 2).

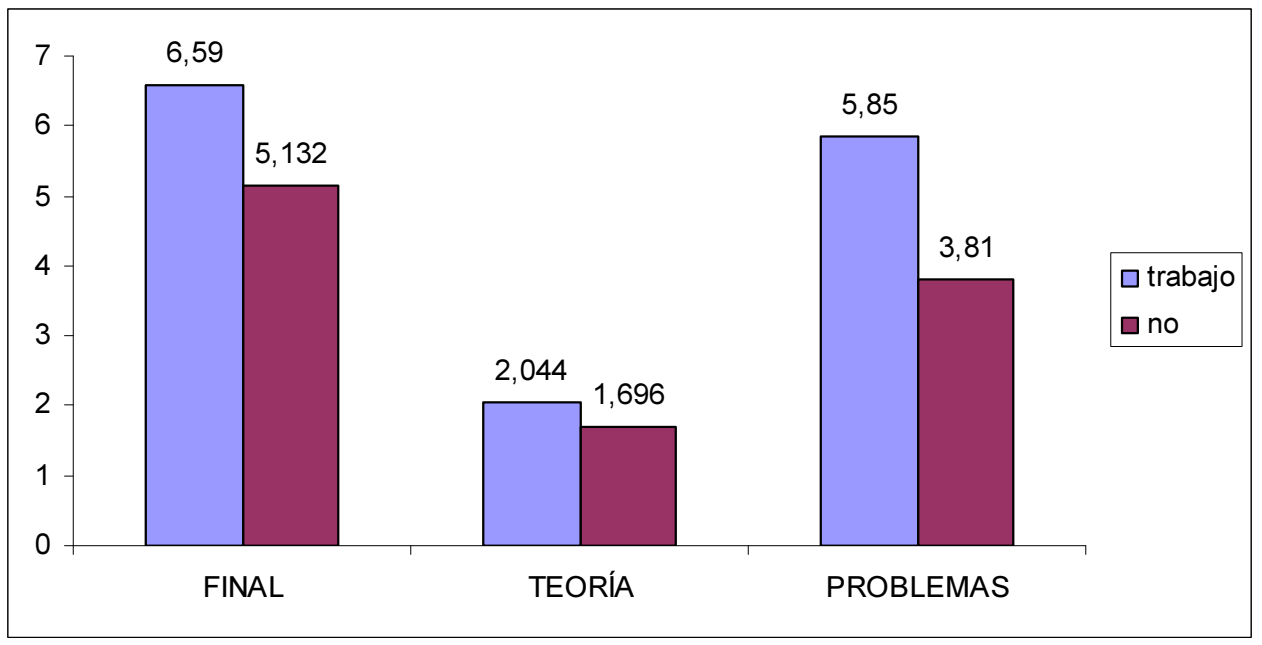

Figura 1. Notas medias obtenidas por los alumnos que han realizado el trabajo (azul) o no (violeta). Se muestran las medias finales, de la parte de teoría y de la parte de problemas 


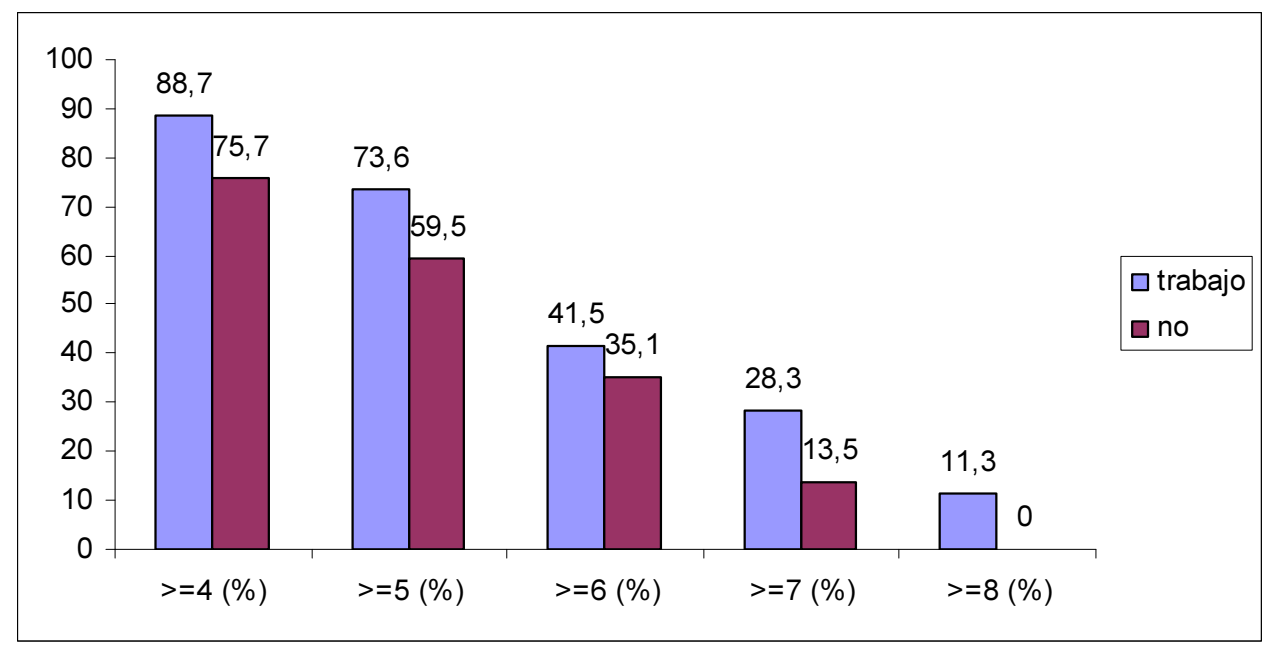

Figura 2. Porcentaje de alumnos con nota mayor o igual a 4,5,6,7 y 8. Se muestran los resultados para el grupo de alumnos que ha realizado el trabajo (azul) o no (violeta).

Tabla 1. Prueba $F$ de fisher para el análisis de la varianza

\begin{tabular}{|l|c|c|c|c|c|c|}
\hline & Final trabajo & Final no trabajo & Pr trabajo & Pr no trabajo & TA trabajo & TA no trabajo \\
\hline Media & 6,59 & 5,13 & 5,85 & 3,41 & 2,04 & 1,70 \\
\hline Varianza & 3,10 & 2,65 & 2,66 & 2,04 & 0,42 & 0,44 \\
\hline Observaciones & 53 & 36 & 53 & 36 & 53 & 36 \\
\hline Grados de libertad & 52 & 35 & 52 & 35 & 52 & 35 \\
\hline $\mathrm{F}$ & 1,17 & & 1,30 & & 0,94 & \\
\hline $\mathrm{P}(\mathrm{F}<=\mathrm{f})$ una cola & 0,32 & & 0,21 & & 0,42 & \\
\hline Valor crítico para F (una cola) & 1,70 & & 1,70 & & 0,61 & \\
\hline
\end{tabular}

Tabla 2. Prueba t de student para la comparación de medias suponiendo varianzas desiguales

\begin{tabular}{|l|c|c|c|c|c|c|}
\hline & Final trabajo & Final no trabajo & Pr trabajo & Pr no trabajo & TA trabajo & TA no trabajo \\
\hline Media & 6,59 & 5,13 & 5,85 & 3,41 & 2,04 & 1,70 \\
\hline Varianza & 3,10 & 2,65 & 2,66 & 2,04 & 0,42 & 0,44 \\
\hline Observaciones & 53 & 36 & 53 & 36 & 53 & 36 \\
\hline Diferencia hipotética de las medias & 0 & & 0 & & 0 & \\
\hline Grados de libertad & 79 & & 81 & & 74 & \\
\hline Estadístico t & 4,01 & & 7,48 & & 2,45 & \\
\hline $\mathrm{P}(\mathrm{T}<=\mathrm{t})$ una cola & $6,84 \mathrm{E}-05$ & & $3,91 \mathrm{E}-11$ & & $8,24 \mathrm{E}-03$ & \\
\hline Valor crítico de t (una cola) & 1,66 & & 1,66 & & 1,67 & \\
\hline $\mathrm{P}(\mathrm{T}<=\mathrm{t})$ dos colas & $1,37 \mathrm{E}-04$ & & $7,82 \mathrm{E}-11$ & & $1,65 \mathrm{E}-02$ & \\
\hline Valor crítico de t (dos colas) & 1,99 & & 1,99 & & 1,99 & \\
\hline
\end{tabular}

\section{3-Auto evaluación de competencias transversales.}

En cuanto a la auto evaluación de CT, se observa que la asignatura podría ser punto de control de la CT "Análisis y resolución de problemas", además de servir para trabajar las CT "Aplicación y pensamiento práctico" y "compresión e integración" (Figura 3).

En cuanto a las CT que podrían trabajarse y evaluarse con los trabajos optativos, se observa que podría servir para trabajar "Conocimiento de problemas contemporáneos", "Aprendizaje permanente", "Planificación y gestión del tiempo" y "Pensamiento crítico" (Figura 4).

(cc) EY-NC-ND 2016, Universitat Politècnica de València

Congreso In-Red (2016) 


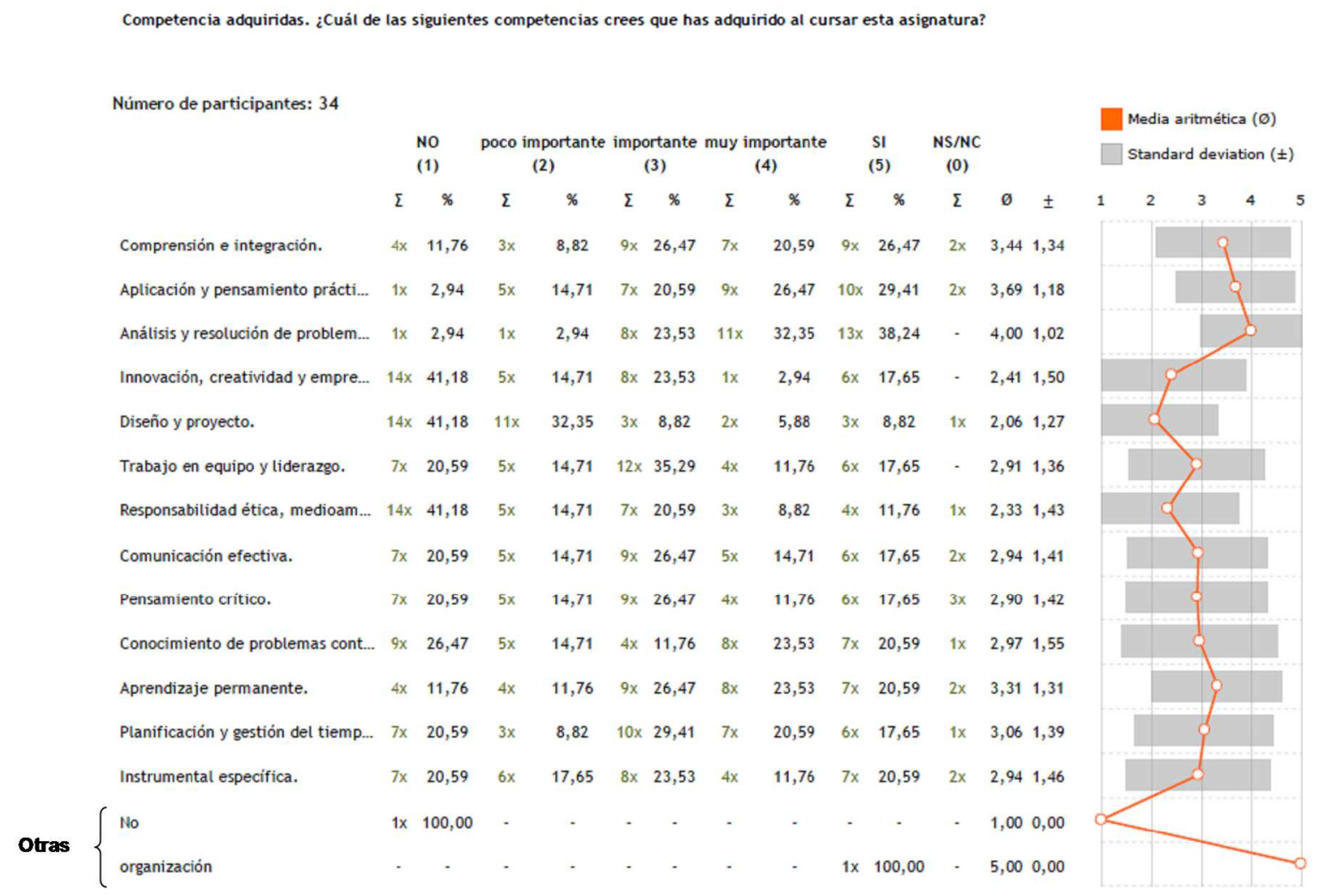

Figura 3. Resultados de la auto evaluación de CT para todos los encuestados, utilizando "online encuesta" (https://www.onlineencuesta.com)

En cuanto a la exposición de los trabajos en clase, ésta parece efectiva para trabajar y evaluar (al menos parcialmente), las CT "Comunicación efectiva" y "trabajo en equipo y liderazgo" (Figura 5).

Cabe destacar que el profesorado detecta las mismas posibilidades de trabajo y evaluación de CT que los alumnos con los trabajos de aplicaciones, especialmente para las CT "Aplicación y pensamiento práctico", "compresión e integración" y "Pensamiento crítico". Se observa además que la exposición de los trabajos es especialmente útil para evaluar el desarrollo de las CT "Comunicación efectiva" y "trabajo en equipo y liderazgo". 


\section{Sin exponer}

$6(60.0 \%)$ : si

4 (40.0\%): no

Número de participantes: 10

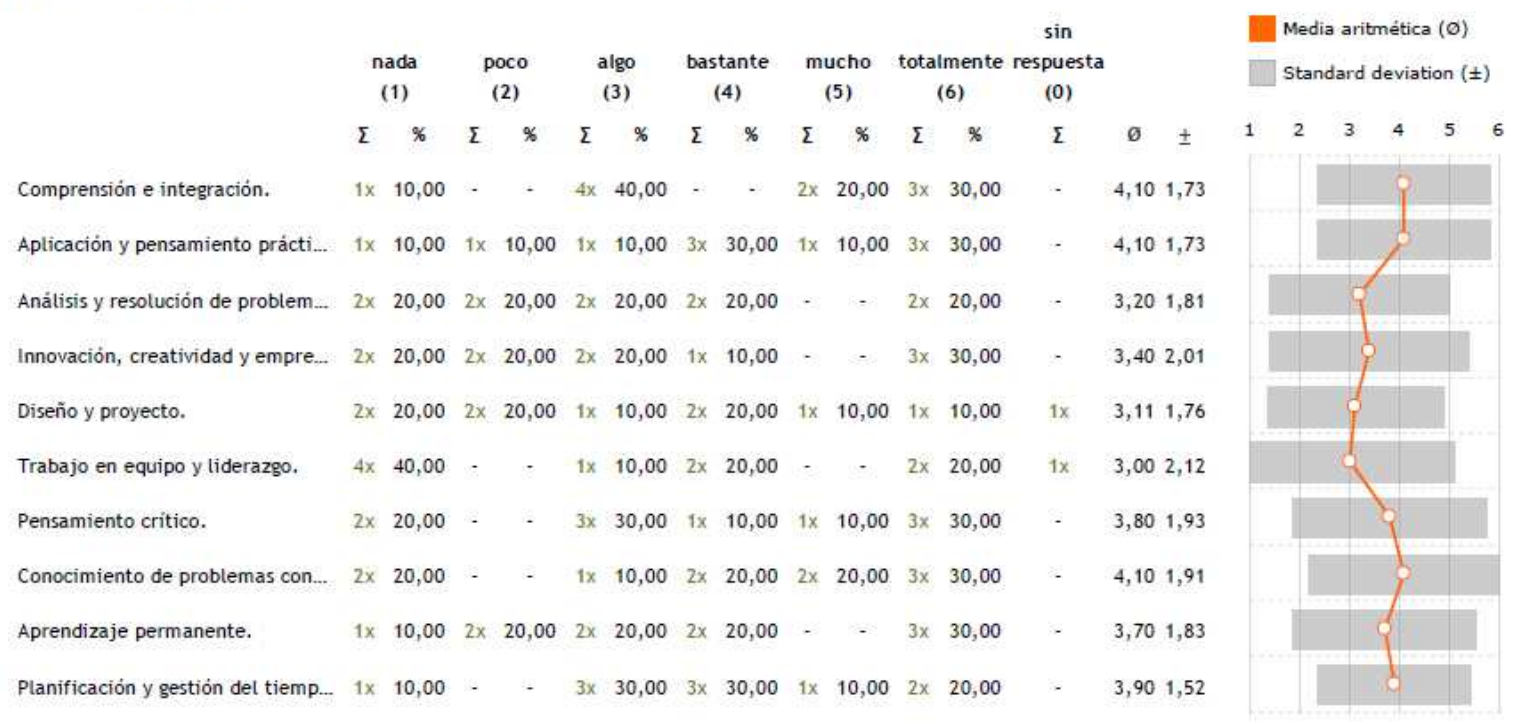

Figura 4. Resultados de la auto evaluación de CT para los encuestados que realizaron el trabajo sin exponerlo, utilizando "online encuesta" (https://www.onlineencuesta.com)

(c) BY-NC-ND 2016, Universitat Politècnica de València 


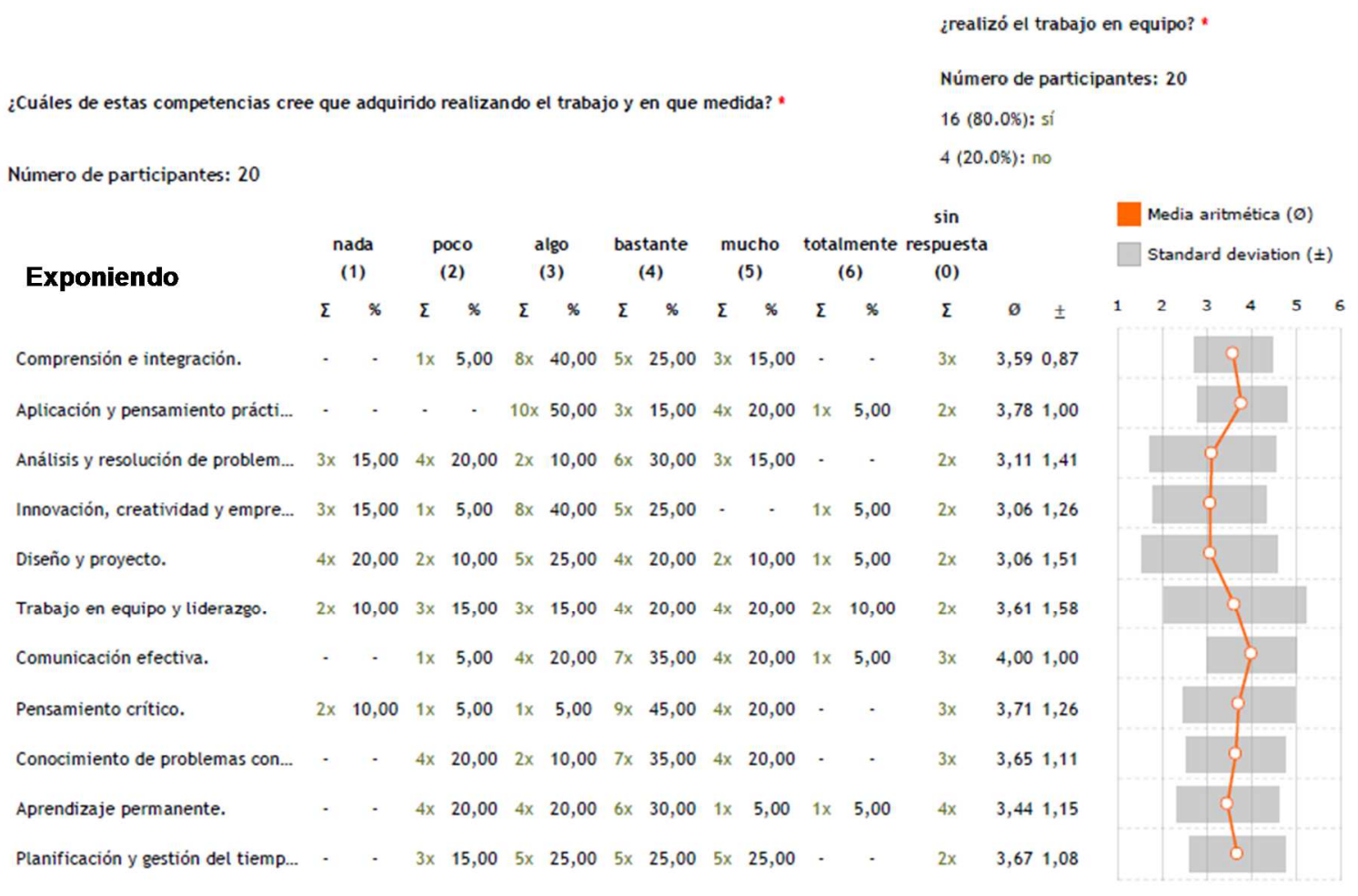

Figura 5. Resultados de la auto evaluación de CT para los encuestados que realizaron el trabajo y lo expusieron, utilizando "online encuesta" (https://www.onlineencuesta.com)

\section{5-Conclusiones}

-Los alumnos que realizaron el trabajo voluntario de aplicaciones de la Química Física a la Tecnología de Alimentos muestran mayor motivación hacia el estudio de la asignatura, lo que se refleja en calificaciones más elevadas en comparación con el resto de alumnos, especialmente en la parte del examen correspondiente a la realización de problemas.

-De acuerdo con la opinión de los alumnos, la asignatura podría ser punto de control, además de la CT "Instrumentales específicas" (para la que ya es punto de control), de la CT "Análisis y resolución de problemas".

-De acuerdo con la opinión de los profesores, los trabajos planteados sirven para el desarrollo y evaluación de varias competencias transversales ("Conocimiento de problemas contemporáneos", "Aprendizaje permanente", y "Pensamiento crítico"). Si los trabajos se exponen en clase en forma de seminarios, sirven además para desarrollar y evaluar las CT "Comunicación efectiva" y "trabajo en equipo y liderazgo". Cabe destacar que para el próximo año la asignatura será también punto de control para la CT "Conocimiento de problemas contemporáneos".

-Los trabajos sobre aplicaciones de la asignatura, además de servir para el aprendizaje activo de los alumnos y para la evaluación de determinadas competencias que no pueden evaluarse en un examen escrito, sirven para que el alumno relacione los conceptos teóricos 
con su futuro campo de trabajo y con la vida real, motivando así al estudio de los conceptos teóricos y la aplicación de los mismos.

\section{6-Referencias}

-ANAYA-DURAND, A. y ANAYA-HUERTAS, C. (2010). "¿Motivar para aprobar o para aprender? Estrategias de motivación del aprendizaje para los estudiantes." en Ciencia Ed. (IMIQ) 25:1

-BAÑOS, J.E. y PÉREZ, J. (2005). "Cómo fomentar las competencias transversales en los estudios de Ciencias de la Salud: una propuesta de actividades." en Educación Médica 8(4): 216-225

-BLANCO-VÁZQUEZ, C. (2006). "Aplicación de la metodología didáctica "PQRST" a la enseñanza de asignaturas complejas o difíciles. Una técnica docente adaptada al nuevo Espacio Europeo de Educación Superior (EEES)." Pharos, 13(1): 13-20

-BLOOM, B.S. (Ed.). ENGELHART, M.D., FURST, E.J., HILL, W.H., KRATHWOHL, D.R. (1956). Taxonomy of Educational Objectives, Handbook I: The Cognitive Domain. New York: David McKay Co Inc.

-CANO-GARCÍA, M.E. (2008). "La evaluación por competencias en la educación superior." en Profesorado. Revista de currículum y formación del profesorado 12, 3

- DE JONG, O. (2006). "Context-based chemical education: how to improve it?" Plenary lecture presented at the 19th International Conference on Chemical Education. Seoul,

Korea, 12-17 August 2006. Disponible en http://www.iupac.org/publications/cei/vol8/0801xDeJong.pdf

- DURÁN-APONTE, E. y DURÁN-GRACÍA, M. (2013). "Aprendizaje cooperativo en la Enseñanza de Termodinámica: Estilos de Aprendizaje y Atribuciones Causales." en Revista Estilos de Aprendizaje 11:11

-FERNÁNDEZ-MARCH, A. (2010). "La evaluación orientada al aprendizaje en un modelo de formación por competencias en la educación universitaria." en Revista de Docencia Universitaria 8(1): 11-34

-FLORES, S. y TREJO, L. (2003). "¿Cómo Mejorar el Proceso Enseñanza - Aprendizaje Mediante la Evaluación - Regulación? El Caso de la Termodinámica." Memorias de las Terceras Jornadas Internacionales de la Enseñanza Universitaria de la Química. La Plata, Argentina. 28 Septiembre al 1 de octubre 2003. Disponible en http://esdocs.com/doc/355805/1-\%C2\%BFc\%C3\%B3mo-mejorar-el-proceso-deense $\% \mathrm{C} 3 \% \mathrm{~B} 1$ anza---unam

-GARCÍA-GARCÍA, M.J. (2009). "Evaluación de Competencias Transversales." Conferencia Facultat d'Informàtica de Barcelona. Universitat Politècnica de Catalunya. 15 de abril de 2009. Disponible en http://www.fib.upc.edu/eees/cicleactivitats_0809/mainColumnParagraphs/05/text_files/file/EvaluacionCompetenciasTransversales.PDF

(c) EY-NC-ND 2016, Universitat Politècnica de València

Congreso In-Red (2016) 
-MAINAR-FERNÁNDEZ, A. M. (2009). "Termodinámica Química: de los Fundamentos a la Aplicación." en Rev. Real Academia de Ciencias. Zaragoza. 64: 7-33

-MENDEZ-COCA, D. (2012). "Motivational Change Realized by Cooperative Learning Applied in Thermodynamics." en European Journal of Physics Education 3(4): 13-26, 2012

- MULOP, N., YUSOF, K. M. y TASIR, Z. (2012). "A review on enhancing the teaching and learning of thermodynamics" en Procedia-Social and Behavioral Sciences, 56, 703712 .

- SOKRAT, H., TAMANI, S., MOUTAABBID M. y RADID M. (2014). "Difficulties of Students from the Faculty of Science with Regard to Understanding the Concepts of Chemical Thermodynamics" en Procedia - Social and Behavioral Sciences, 116, 21: 368372 .

-TABER, K.S. (2015). "Epistemic relevance and learning chemistry in an academic context" en EILKS, I. y HOFSTEIN A. (Eds.). (2015). Relevant Chemistry Education From Theory to Practice. Rotterdam: Sense Publishers.

-UPV: Preguntas frecuentes sobre competencias transversales. 2015. (https://www.upv.es/entidades/ICE/info/U0702443.pdf) 